\title{
Quantum Critical Point in Heavy Fermions
}

\author{
Mucio Amado Continentino \\ Instituto de Física, Universidade Federal Fluminense \\ Campus da Praia Vermelha, Niterói, 24210-340, RJ, Brazil
}

Received on 28 September, 2004

\begin{abstract}
The concept that heavy fermions are close to a quantum critical point and that this proximity determines their physical behavior has opened new perspectives in the study of these systems. It has provided a new paradigm for understanding and probing the properties of these strongly correlated materials. Scaling ideas were important to establish this approach. We give below a brief and personal account of the genesis of some of these ideas 15 years ago, their implications and the future prospects for this exciting field.
\end{abstract}

\section{Introduction}

The problem of heavy fermions has its roots in the field of valence-fluctuations in $f$-electron systems $[1,2]$. From the point of view of theory, it is a natural extension of the work by Mott, Friedel and Anderson [3] on impurities in metals for the case of a lattice of impurities, the Anderson and Kondo lattices [4]. These strongly correlated materials are intermetallic compounds containing unstable $f$-shell elements, as ytterbium $(Y b)$, cerium $(C e)$ and uranium $(U)$. Since the $f$-ions are disposed on the sites of a lattice, they have lattice translation invariance and ideally their resistivity should vanish as temperature approaches zero.

Heavy fermions (HF) are physical realizations of the Landau Fermi liquid since the effect of the strong interactions among the quasi-particles is essentially to renormalize the parameters in a simple Fermi liquid description. Below a characteristic temperature, heavy fermions show all the features of a Fermi liquid, a linear temperature dependent specific heat, a Pauli susceptibility and a resistivity that varies as $T^{2}$ and all with strongly renormalized coefficients [4]. For example, the mass of the quasi-particles in $\mathrm{CeCu}_{6}$, obtained from the coefficient of the linear temperature dependent term of the specific heat, is of the order of thousand times larger [4] than that of electrons in a normal metal as copper $(\mathrm{Cu})$. Then, the term heavy fermion is indeed appropriate. It is remarkable that in spite of this huge renormalization, the Fermi liquid picture still applies. This behavior is in strong contrast with that of high-temperature superconductors where the combination of strong interactions and low dimensionality produces profound modifications on the physical properties.

\section{The phase diagram}

The physics of heavy fermions is due to two main effects. The Kondo effect with a tendency to screen the moments and produce a non-magnetic ground state and the RKKY interaction [4] which favors long range magnetic order. The result of this competition is summarized in the Doniach phase diagram of the Kondo lattice model Hamiltonian [5, 6]. This diagram has a quantum critical point (QCP) at a critical value of the ratio $(J / W)_{c}$ where $J$ stands for the interaction among the localized and conduction electrons and $W$ is the bandwidth of the latter [5, 6]. For small values of $(\mathrm{J} / \mathrm{W})$ there is a long range ordered magnetic phase, in general antiferromagnetic and beyond the QCP, for $(J / W)>(J / W)_{c}$, a non-magnetic ground state where the Kondo effect prevails (Fig. 1).

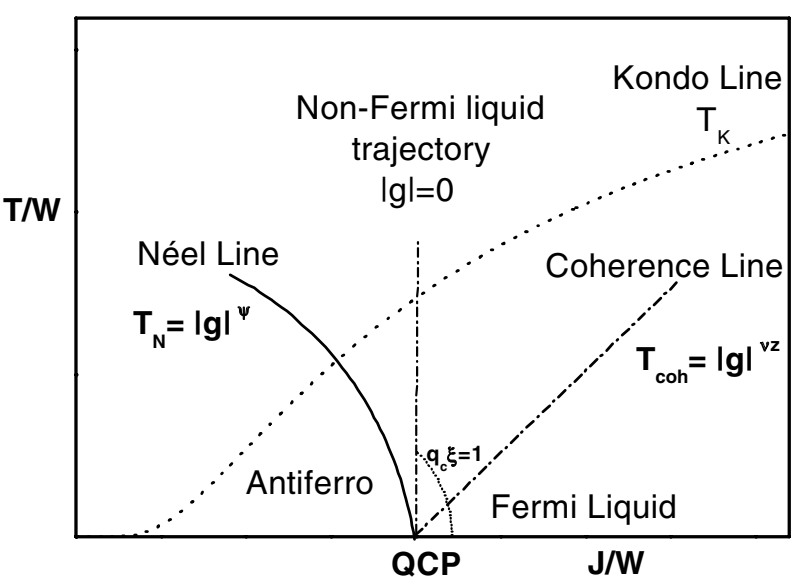

Figure 1. Phase diagram of the Kondo lattice showing the coherence line and the non-Fermi liquid trajectory. The curve $q_{c} \xi=1$ is a dimensional crossover line, from quantum local on the right, to quantum $(\mathrm{d}+\mathrm{z})$ criticality on the left of this curve.

In the magnetically ordered region, at finite temperatures, there is a line of Néel transitions where long range magnetic order is destroyed by thermal fluctuations $(d>2)$ (Fig. 1).

The discovery of superconductivity [7], a phase not contemplated in Doniach's diagram and the crossover to the renormalized Fermi liquid regime below a characteristic temperature $T_{c o h}$, much lower than the single ion Kondo temperature $T_{K}$, were among the challenging features observed in heavy fermions. This low temperature scale shows up in thermodynamic [8] and transport experiments 
[9] and was soon recognized as a phenomenon specific of the Kondo lattice. It involves a collective behavior of the moments and, as such, is unrelated to the single impurity Kondo physics. The existence of two energy scales in heavy fermions is nicely illustrated by transport measurements in $\mathrm{CeCu}_{6}$ shown in Fig. 2. The high temperature scale in the incoherent regime is the Kondo temperature $T_{K}$. The coherence temperature $T_{c o h}$ in this experiment appears associated with the drop in resistivity at low temperatures, as coherence sets in among the scatterers.

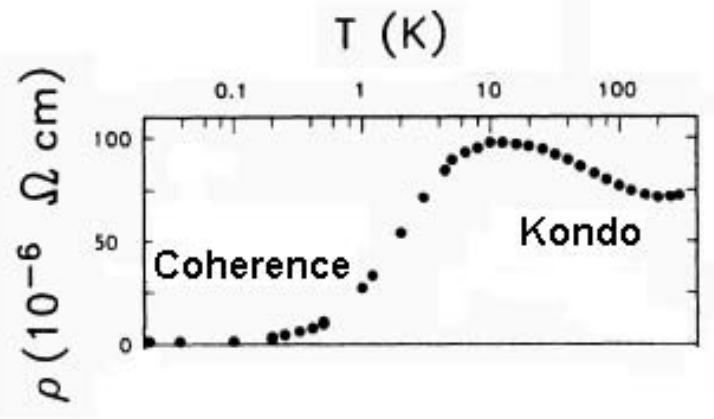

Figure 2. Resistivity of $\mathrm{CeCu}_{6}$. The logarithmic rise below the minimum at $\approx 120 \mathrm{~K}$ is associated with an incoherent single ion Kondo effect. Below the maximum, as coherence sets in among the scatterers, the resistivity drops. At very low temperatures the resistivity rises with a $T^{2}$ power law (adapted from Ref. [9]).

\section{Scaling theory}

In 1988, the Kondo effect was already a very well understood phenomenon [10]. However, for the coherence temperature it was lacking a proper theoretical description. The reason was the complexity of the lattice problem formulated either as a periodic Anderson model or as a Kondo lattice, which demanded drastic approximations that, at most, were adequate to treat the mixed-valency regime. Invariably, these missed the magnetic transition and led to the appearance of the single ion Kondo temperature, with its characteristic exponential dependence on $(J / W)$, as the relevant energy scale also for the non-magnetic Kondo lattice [11]. When we started working on heavy fermions that year, we were shifting our main interest from random magnetic systems to the area of strongly correlated electrons. We had been involved with the question of the existence of a field dependent critical line in spin glasses [12], which made us acquainted with renormalization group (RG) studies and scaling theories of these and random field models. One approach considered a description of random field Ising systems in terms of a zero temperature fixed point which was stable along the temperature axis [13]. This unusual renormalization group flow with temperature acting as an irrelevant variable, in the RG sense, gave rise to the effect of dimensional reduction. We started to discuss a scaling theory of heavy fermions considering the existence of a zero temperature fixed point associated with the critical point $(J / W)_{c}$ of Doniach's diagram [14]. In this case temperature could not be an irrelevant variable as this would imply the critical behavior along the Neel line being governed by the zero temperature fixed point. While in a classical system, as the random field Ising ferromagnet, this can be realized and gives rise to anomalous dynamics [13], it could not clearly be the case for a quantum system. However, if temperature is relevant at a zero temperature fixed point, instead of dimensional reduction we would expect to have an increase in the effective dimensionality of this fixed point. The identification of the exponent which renormalizes temperature and determines the effective dimension as being the dynamic exponent $z$, which scales time, turned out as a natural consequence of the Heisenberg uncertainty relation. Furthermore, if the effective dimension $d_{e f f}=d+z$ is larger than the upper critical dimension $d_{c}$, which for the antiferro-paramagnetic transition in the $\mathrm{KL}$ is $d_{c}=4$, then the exponents associated with the zero temperature fixed point should be mean field like. This kind of reasoning using simple scaling ideas led us $[14,15,16]$ to reach many of the conclusions of the works of Hertz [17] and Young [18] on quantum phase transitions.

An expansion of phenomenological renormalization group equations close to the zero temperature fixed point of the Kondo lattice allowed to obtain the scaling form of the free energy density [14, 15],

$$
f_{s} \propto|g|^{2-\alpha} F\left[\frac{T}{|g|^{\nu z}}, \frac{h}{|g|^{\beta+\gamma}}\right]
$$

where $g=(J / W)-(J / W)_{c}$ measures the distance to the quantum critical point in Doniach's diagram and $h$ is a field conjugated to the order parameter of the magnetic phase, in most cases a staggered field. The scaling function is such that, $F[0,0]=$ constant. The exponents $\alpha, \beta$ and $\gamma$ are usual critical exponents associated with a phase transition and are related by the standard scaling relation, $\alpha+2 \beta+\gamma=2$. The hyperscaling relation however is modified since the relevant fixed point is at zero temperature. Due to the quantum character of the phase transition it is given by [14],

$$
2-\alpha=\nu(d+z)
$$

Eq. 1 has two remarkable features. First the appearance of the dynamic exponent $z$ in the expression for the free energy. This is going to show up in all thermodynamic quantities which are obtained as derivatives of Eq. 1. This is a direct consequence of the quantum character of the transition which inextricably couples statics and dynamics. Also, as a bonus, temperature appears scaled by a crossover temperature, $T^{*} \propto|g|^{\nu z}$ associated with the quantum critical point and that remains to be interpreted. This new energy scale, characteristic of the lattice had all the features to be recognized as the so-called coherence temperature which appears in the thermodynamic experiments. I quote from our 1989 paper [14]; We identify the crossover line with the so-called "coherence transition" observed in heavy fermions and which marks the onset of the dense Kondo regime with decreasing temperature.

The identification $T^{*} \sim T_{\text {coh }}$ represents a crucial step in the scaling approach and is full of consequences. Since $T_{c o h}$ 
marks the onset of the dense Kondo or renormalized Fermi liquid regime in the non-critical side of the phase diagram ( $\left.J / W>(J / W)_{c}\right)$, the free energy for $T \ll T_{c o h} \propto|g|^{\nu z}$ has a Sommerfeld expansion in even powers of the scaled temperature. This is given by

$$
f_{s} \propto|g|^{2-\alpha}\left[1+\left(\frac{T}{T_{c o h}}\right)^{2}+O\left(\left(\frac{T}{T_{c o h}}\right)^{4}\right)\right]
$$

to order $\left(T / T_{c o h}\right)^{2}$. From this expression we can obtain the coefficient of the linear term of the specific heat in the Fermi liquid regime,

$$
C / T \propto|g|^{2-\alpha-2 \nu z}=|g|^{\nu(d-z)}
$$

where we used the quantum hyperscaling relation in the last step [14].

The relevance of a scaling approach for heavy fermions depends of course whether these systems are close to a QCP. That this is the case is shown by the fact that small pressures either positive or negative can drive these systems from a magnetic to a non-magnetic ground state or vice-versa. Now from Eq. 4 we had the possibility of understanding the large masses of heavy fermions in terms of proximity to a QCP, if the dynamic exponent satisfies the inequality $z>d$. This was also an exciting result since, for the first time, we were able to identify the exponents governing the mass enhancement of a many-body system in terms of the critical exponents of a quantum phase transition.

Soon, it became clear to us [15] that the trajectory where the system is sitting at the critical point $(|g|=0)$ was a special one. In this case one tunes the system to the QCP, i.e, to $|g|=0$, by pressure, doping, or magnetic field and varies the temperature. Along this path the system never crosses the coherence line and consequently does not enter the Fermi liquid regime (see Fig. 1). Then, on this special trajectory we expect to find non-Fermi liquid behavior [15] down to the lowest temperatures. The behavior of a given physical quantity along this line is obtained by demanding that the dependence of its scaling function on the scaled variables cancels its overall dependence on the distance $|g|$ to the quantum critical point. For the specific heat at the QCP this yields,

$$
C / T \propto T^{\frac{d-z}{z}}
$$

which in general is clearly a non-Fermi liquid behavior. Applying the same type of argument for the order parameter susceptibility, we find at the QCP $(|g|=0)$,

$$
\chi \propto T^{-\frac{\gamma}{\nu z}} .
$$

and for the correlation length

$$
\xi \propto T^{-\frac{1}{z}} .
$$

The scaling approach $[14,15,16]$ as we have proposed in the years from 1989 to 1993 goes beyond the KL model and holds in general for other types of zero temperature phase transitions in many-body systems. It does not necessarily require the existence of an order parameter and was easily extended to treat the Mott transition [19]. The paper by Fisher et al. [20] on quantum phase transitions in bosonic systems appeared soon after our scaling theory of heavy fermions. Some concepts introduced in this paper could be straightforwardly carried out to the electronic problem. For example, the notion of two kinds of superfluidinsulator transitions was immediately extended for metalinsulator transitions [19]. It turned out very useful to distinguish between density-driven and interaction-driven Mott transitions in the Hubbard model as they are in general in different universality classes [19]. The scaling approach also allowed to obtain the upper critical dimension for the interaction-driven Mott transition and make an interesting conjecture on the nature of the density-driven one [19].

\section{Breakdown of hyperscaling}

Originally, we have supplemented the scaling approach with the assumption generally known as the extended scaling hypothesis or hyperscaling. It consists in identifying the shift exponent $\psi$ of the critical line of finite temperature phase transitions, $T_{N} \propto|g|^{\psi}$, with the exponent $\nu z$ of the crossover line [14], i.e., $\psi=\nu z$. This assumption which is justified below the upper critical dimension does not hold for $d+z>d_{c}$ as discussed below.

The notion that hyperscaling breaks down due to a dangerous irrelevant variable above the upper critical dimension appears in the context of quantum phase transitions at least in two different situations. The first addresses us to the work of Millis [21] which extends Hertz approach of quantum phase transitions in metals [17] for the case of finite temperatures. Because temperature scales close to the QCP with the dynamic exponent $z$, i.e., $T^{\prime}=b^{z} T$ ( $b$ is the scaling factor) and the quartic interaction in the generalized LandauGinzburg-Wilson functional scales as $u^{\prime}=b^{4-(d+z)} u$, the combination $u T$ in fact behaves as a relevant field [21] $(d<4)$. Integration of the renormalization group equations shows that the correlation length diverges along a line of finite temperature phase transitions, $T_{N} \propto(|g| / u)^{\psi}$, with the shift exponent given by, $\psi=z /(d+z-2)$. Then, in spite that $u$ is an irrelevant variable that scales to zero at the $T=0$ Gaussian fixed point for $d+z>4$, it breaks the hyperscaling relation $\psi=\nu z$.

Another consequence of the dangerous irrelevancy of the interaction $u$, for $d+z>4$, is that the correlation length at the QCP will diverge with temperature according to a different power law [21] from that obtained in Eq. 7 using naive scaling. This is given by [21] $(\nu=1 / 2)$,

$$
\xi \propto \frac{1}{\sqrt{u}} T^{-\frac{\nu}{\psi}} .
$$

The dangerous character of $u$ is clearly reflected on the fact that it appears on the denominator of this equation.

The effect of $u$ on thermodynamic quantities requires a knowledge of the dependence of the free energy on this interaction and this brings us to the next situation of violation of hyperscaling [25]. The idea is to iterate the renormalization group equations $n$ times with a scaling factor $b$, until $g\left(\ell=b^{n}\right) \gg 1$ and fluctuations become negligible [22]. At 
this point we can use Landau expansion of the free energy in terms of the order parameter $M$,

$$
f_{L}(\ell)=\frac{1}{2} g(\ell) M^{2}(\ell)+u(\ell) M^{4}(\ell)
$$

and follow the usual method for dealing with this equation [23] to obtain, $M=\sqrt{g(\ell) / u(\ell)}$ and $f_{L}(\ell)=|g(\ell)|^{2} / u(\ell)$. Finally, using the scaling results for the variables involved, we get,

$$
f_{L} \propto \ell^{-(d+z)} \frac{\left|\ell^{2} g(T)\right|^{2}}{u \ell^{4-d-z}}=\frac{\left|g-u T^{1 / \psi}\right|^{2}}{u}
$$

for $d+z>4$ with $g=g(T=0)$. Notice that at $T=0$, $f_{L} \propto|g|^{2} / u$ and as $|g| \rightarrow 0$ this mean-field contribution is dominant over the Gaussian one, $f_{G}(T=0) \propto|g|^{\nu(d+z)}$ for $d+z>4$. Consequently, the exponent $\alpha$ of the singular part of the zero temperature free energy density defined in Eq. 1 remains fixed at the mean-field value, $\alpha=0$, for all $d+z>4$. This implies a violation or breakdown of the quantum hyperscaling relation, Eq. 2 , for $d+z>4$.

Then, the dangerous irrelevant variable $u$ is essential even at $T=0$ to yield the correct mean-field critical behavior of the order parameter, $M \propto|g|^{1 / 2}$ and its susceptibility, $\chi \propto|g|^{-1}$. Besides at $|g|=0$ and $T=0$, we get that $M \propto h^{1 / 3}$ for the order parameter in the presence of the conjugate field $h$. Thus, at zero temperature, for $d+z>4$, due to the dangerous irrelevant nature of $u$, the critical exponents assume the mean-field values, $\beta=1 / 2, \gamma=1$ and $\delta=3$ instead of the dimensional dependent Gaussian exponents [25] which appear if $u$ is not taken into account [23].

Also at the QCP we find that the order parameter susceptibility, $\chi(|g|=0, T) \propto T^{-\gamma / \psi}$, with $\gamma=1$, instead of Eq. 6.

When further approaching the critical line $g-u T_{N}^{1 / \psi}=$ 0 , at $T \neq 0$, there is an important Gaussian contribution to the free energy, $f_{G} \propto A(T)\left|g-u T^{1 / \psi}\right|^{2-\tilde{\alpha}}$, such that, $2-\tilde{\alpha}=\tilde{\nu} d$ where $\tilde{\alpha}$ and $\tilde{\nu}=1 / 2$ are Gaussian thermal exponents and [21] $A(T) \propto T^{(\tilde{\alpha}-\alpha) / \nu z} \propto T$ (see also Ref. [24]). It is easily seen that for $T \neq 0$, this contribution dominates the mean-field one given by Eq. 10 .

It is remarkable that at the QCP $(|g|=0)$, the specific heat given by Eq. 5 which at this point can be identified with the purely Gaussian result $(u=0)$ is more singular than that obtained from Eq. 10, namely, $C / T \propto u T^{2(d-2) / z}$. This in turn is more singular than, $C / T \propto u^{d / 2} T^{(d+z)(d-2) / 2 z}$, obtained from $f_{G}$ above, for all $d+z>4$.

This purely Gaussian term, Eq. 5, however is insensitive to the critical line of thermal phase transitions that appears as a correction in the quartic interaction $u$. Since the QCP is a special point of this line one must be careful when dealing with this contribution [26]. The analysis of experimental data on thermodynamic quantities close to a QCP is not a simple task. There are contributions to the free energy with unknown pre-factors that become dominant in different intervals of temperature as $\left|g-u T^{1 / \psi}\right|$ is reduced and as the QCP is approached. It is not trivial, for example, to disentangle the thermal and quantum contributions to the specific heat when $T_{N}$ is small but finite [27].
Finally, it is important to point out that if $\psi=\nu z$, the results above at $|g|=0$ reduce to those obtained using naive scaling [24].

\section{Local quantum criticality}

Our next goal after proposing the scaling theory was to determine the universality class of the heavy fermion quantum critical point from experimental data. The way to move in Doniach's phase diagram is to apply pressure in the system in order to vary the ratio $(J / W)$. In 1989 , the material which was more systematically studied as a function of pressure was $\mathrm{CeRu_{2 }} \mathrm{Si}_{2}$. This work was mostly carried out in Grenoble [28]. This heavy fermion material is nonmagnetic and consequently located to the right of the QCP in Fig. 1. Collecting the available data on this system, we were able to determine relations among the critical exponents of $\mathrm{CeRu}_{2} \mathrm{Si}_{2}$. These relations [15], $2-\alpha=\nu z=\phi_{h}$ were later found to also apply to other systems where we used a similar type of analysis [16] (see Fig. 3). The exponent $\phi_{h}$ is that which scales the uniform magnetic field $H$ in the argument of the free energy scaling function, i.e., $H$ appears in this function as, $H /|g|^{\phi_{h}}=H / H_{c}$ [15]. In particular, the relation $2-\alpha=\phi_{h}$ implies that $m=\partial F / \partial H=f\left(H / H_{c}\right)$, such that, $m\left(H_{c}\right)=$ constant for all pressures and this was observed in the experiments $[15,28]$. The reason the exponents themselves could not be directly obtained for $\mathrm{CeRu_{2 }} \mathrm{Si}_{2}$ is that there is no positive critical pressure that would allow to define a scaling variable $|g| \propto\left|P-P_{C}\right|$.

The relation, $2-\alpha=\nu z$ represented a real puzzle. Eq. 2 shows that this is just the quantum hyperscaling relation with $d=0$. Zero dimensionality is associated with local effects and seemed quite incompatible with any form of criticality.

The solution to the puzzle came a few years later [29]. It is connected to the quantum character of the critical point and can be explained in terms of local criticality [30]. In quantum phase transitions the effective dimension is $d_{e f f}=$ $d+z$. The $d$ of course refers to the spatial dimensions while the $z$ concerns extra dimensions in time directions. Then, it is possible that the correlation length in the time directions, $\tau=\xi^{z}$ is already large while that in the space directions is still of the order of an interatomic distance. For all purposes, in this region of the phase diagram, from the point of view of critical behavior the system has an effective dimensionality $d_{e f f}=z$ and spatial dimension $d=0$.

However, this local criticality corresponds to just a regime in the neighborhood of the QCP. As the system gets even closer to the quantum phase transition, the correlation length grows and there is a crossover to true $(\mathrm{d}+\mathrm{z})$ criticality, as shown in Fig. 1. Then, the conclusion is that the systems we have investigated and satisfy the relations $2-\alpha=\nu z=\phi_{h}$ are close but not too close to the QCP in a region of the phase diagram where correlations exist mainly in the time directions. 


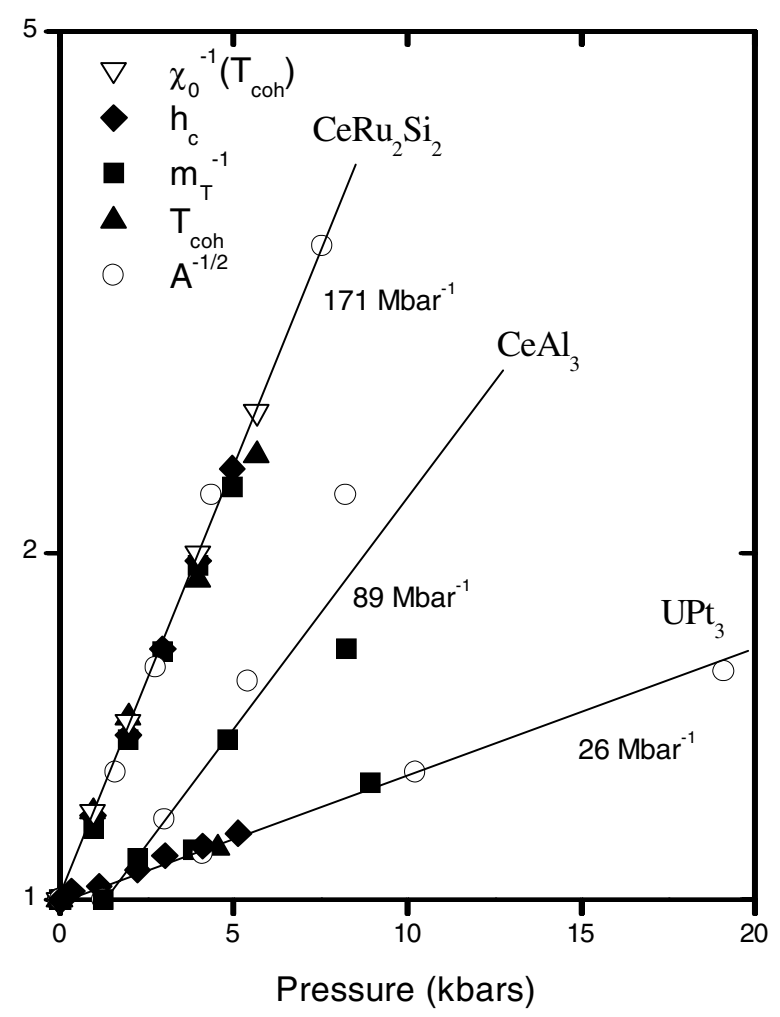

Figure 3. Semi-logarithmic plot $X(P) / X\left(P_{0}\right)$ for several physical quantities $X$, at or below $T_{c o h}$, as a function of pressure for different heavy fermions. For $\mathrm{CeAl}_{3}, P_{0}=1.2$ kbars otherwise $P_{0}=0$. The numbers close to the lines are their inclinations which correspond to the values of the Grüneisen parameters (see Ref. [16] and references therein). The fact that the experimental points fall in a line for a given system, implies the relations $2-\alpha=\nu z=\phi_{h}$ among the critical exponents. This in turn shows that these systems at their reference pressures $P_{0}$ are locally critical and positioned to the right of the curve $q_{c} \xi=1$ in the phase diagram of Fig. 1.

This local regime can be easily identified [29] in Gaussian theories of heavy fermions [31] with dynamic exponent $z=2$. The local description requires the coefficient of the $k^{2}$ term in the Gaussian free energy to be small but different from zero [29]. Otherwise, we have to consider the possibility of quantum Lifshitz points [32].

Along the line $q_{c} \xi=1$ in the phase diagram of Fig. 1 the correlation length is constant. In this case $\xi=q_{c}^{-1}=\pi / a$ where $q_{c}^{-1}=\pi / a$ is a cut-off in momentum space and $a$ the interatomic distance. The local regime appears to the right of this line, for $q_{c} \xi<1$, where the correlation length is of the order or smaller than an interatomic distance. In this local regime there is a single energy scale, the coherence temperature, which is related to the inverse of the characteristic time, $k_{B} T_{c o h}=\hbar \tau^{-1}=|g|^{\nu z}$. This regime is characterized by $\omega / T$ scaling [33], a pressure independent KadowakiWoods ratio [34], a Wilson ratio equal $3 / 2$ and a coefficient $A$ of the $T^{2}$ term of the resistivity which is related to $T_{c o h}$ by, $A \propto 1 / T_{c o h}^{2}$ (see Ref. [29]). In spite of its local character, the regime $q_{c} \xi<1$ is truly critical in the sense that the characteristic time (length), $\hbar \tau^{-1}=|g|^{\nu z}$ reflects the proximity to the quantum phase transition. The line $\xi(T, g)=1 / q_{c}$ can be viewed as a dimensional crossover line where the system crosses from $z$ to $d+z$ behavior as it approaches the QCP.

\section{Prospects}

Much progress has been made in the last years on the study of heavy fermions [35]. The paradigm of quantum criticality resulted in a useful approach which has revealed many unexpected features in these systems. Since the observation of non Fermi liquid behavior at the quantum critical point [36], this has remained a special region of interest. Many systems have been discovered which display quantum criticality. Although the picture which emerges from the experiments is in general agreement with the scenario described above, there remain some important differences with the theoretical predictions. A puzzling one concerns the dimension of the critical fluctuations. The shape of the critical line, the temperature dependence of the specific heat and the behavior of the resistivity are consistent in many heavy fermions with a regime of two-dimensional fluctuations which is reluctant to cross over to the expected $3 d$ (or $3+z$ ) criticality. At $|g|=0$ the slow divergence of the correlation length with temperature controlled by an exponent $\nu / \psi<1$, as in Eq. 8, could be responsible for this behavior. This slow divergence also implies that the local regime extends to the close neighborhood of the QCP, as the condition $q_{c} \xi \gg 1$ seems hard to attain in the non-critical side of the phase diagram.

The scaling theory of quantum criticality has a limited space of free parameters. In $3 d$ as long as the effective dimension $d_{\text {eff }}=d+z$ is above the upper critical dimension, knowledge of the dynamic exponent $z$ is sufficient to determine all the thermodynamic quantities in the neighborhood of the QCP. This is independent of a particular microscopic theory and relies just on scaling which in turn depends on very few universal assumptions. While one can think of this as a tyranny of the quantum critical point [37], it may be viewed as an unique opportunity to reach a detailed understanding of some very basic physics [24, 38].

Disorder is a real ingredient in heavy fermions [39]. This may raise the upper critical dimension, give rise to new universality classes and to Griffiths singularities [40]. However for all this to occur, at least in a $3 d$ system, disorder must be very strong. Most probably in clean systems this is not the source of incompatibilities between experimental results and the scaling theory.

On the other hand it is conceivable that fluctuation induced, weak first order transitions modify the character of the critical line close to the QCP and give rise to inhomogeneous behavior at microscopic scales [41]. Such inhomogeneities have been recently reported in several heavy fermions [42] near a QCP and at least in $\mathrm{CeIn}$ they are clearly associated with a first order transition [43]. They would certainly have a considerable impact on the physical properties and on the quantum critical point scenario, although for weak first order transitions scaling may still apply [41]. The situation is still more interesting and complex since superconductivity appears commonly near antiferromagnetic 
quantum critical points, even in systems with phase separation [42]. We have shown recently that the coupling between superconducting and antiferromagnetic critical fluctuations may change the order of the magnetic quantum phase transition giving rise to a weak first order transition at zero temperature [44]. In this case it is this very coupling which is ultimately responsible for inhomogeneities.

An interesting line of study on quantum criticality in metals has been to investigate the changes in the nature of the elementary excitations of the long range ordered magnetic phase, below the critical line, as the QCP is approached from the left [45] (see Fig. 1). One way of probing these excitations is through the electrical resistivity since they scatter the conduction electrons. Then the transport measurements allow to accompany the changes in the spin-wave parameters as the distance $|g|$ to the QCP is reduced, for example, by applying pressure on the material [45].

Many routes are open for the study of quantum criticality: detailed experiments, new materials with different types of criticality including ferromagnetic QCP, proper discrimination between transition metals [46] and heavy fermion quantum critical behavior and superconductivity [47]. The exploration of the phase diagram with different control parameters as a magnetic field [48] adds an extra dimension to the problem. Also with further increasing pressure we may return to the valence fluctuation problem now with another perspective [37]. At this point we can appreciate the progress that has already been made and at the same time anticipate a future with challenging problems and possibly unexpected discoveries in experiments and theory.

\section{Acknowledgments}

I would like to thank many colleagues with whom I had discussions and correspondence in the area of strongly correlated materials during these years. In particular, my special thanks to Amós Troper with whom I started to work on this subject, to Enzo Granato, A. Eichler and A. S. Ferreira for a critical reading of the manuscript. I wish to thank the Brazilian agencies $\mathrm{CNPq}$ and FAPERJ for their support. This work has been performed in the context of the projects PRONEX-CNPq-FAPERJ/171.168-2003, PRONEX98/MCT-CNPq-364.00/00 and FAPERJ/Cientista do Nosso Estado programs.

\section{References}

[1] J. M. Lawrence, P. S. Riseborough, and R.D. Parks, Rep. Prog. Phys. 44, 1 (1981); Valence Instabilities and Related Narrow-Band Phenomena, Ed. R. Parks, Plenum Press, NY, 1977; Valence Instabilities, Eds. P. Wachter and H. Boppart, North-Holland, The Netherlands, 1982; see also Proceedings of the 6th International Conference on Valence Transitions, Eds. G. E. Barberis, M. Foglio, J. E. Crow and P. Schlottmann, North-Holland, 1991.

[2] J. G. Sereni, J. Physics F-Metal Physics, 10, 2831 (1980); J. G. Sereni, G. L. Olcese, and C. Rizzuto, J. de Physique 40, 337 Suppl. 5 (1979)

[3] see P. W. Anderson, Phys. Rev. 124, 41 (1961) and references therein.
[4] J. D. Thompson and J. M. Lawrence in Handbook on the Physics and Chemistry of Rare Earths, Lanthanides / Actinides:Physics-II, edited by K.A. Gschneider Jr., L. Eyring, G.H. Lander, and G.R. Choppin, Elsevier Science B.V., Chapter 133 (19), 383 (1994).

[5] S. Doniach, Physica B91, 231 (1977); C. Lacroix and M. Cyrot, Phys. Rev. B 20, 1969 (1979); R. Jullien, J. N. Fields, and S. Doniach Phys. Rev. B 16, 4889 (1977); R. Jullien, J. Fields, and S. Doniach Phys. Rev. Lett. 38, 1500 (1977).

[6] P. Pfeuty, R. Jullien, and K. A. Penson in Real Space Renormalization Group, Springer Verlag, Berlin, 119 (1982); R. Jullien, Can. J. Phys. 59, 605 (1981).

[7] F. Steglich, J. Aarts, C. D. Bredl, W. Lieke, D. Meschede, W. Franz, and H. Schäfer Phys. Rev. Lett. 43, 1892 (1979); U. Rauchschwalbe, W. Lieke, C. D. Bredl, F. Steglich, J. Aarts, K. M. Martini, and A. C. Mota, Phys. Rev. Lett. 49, 1448 (1982).

[8] Z. Fisk, H. R. Ott, T. M. Rice, and J. L. Smith, Nature 320, 124 (1986); J. Flouquet, P. Haen, C. Marcenat, P. Lejay, A. Amato, D. Jaccard, and E. Walker, J. Magn. Magn. Mater. 52, 85 (1985); G. R. Stewart, Z. Fisk, and M. S. Wire, Phys. Rev. B30, 482 (1984); M. Niksch, B. Lüthi, and K. Andres, Phys. Rev. B22, 5774 (1980); G. R. Stewart Rev. Mod. Phys. B56, 755-787 (1984); G. Aeppli, H. Yoshizawa, Y. Endoh, E. Bucher, J. Hufnagl, Y. Onuki, and T. Komatsubara, Phys. Rev. Lett. B57, 122 (1986).

[9] T. Penney, F. P. Milliken, S. von Molnar, F. Holtzberg, and Z. Fisk, Phys. Rev. B34, 5959 (1986).

[10] K. G. Wilson, Phys. Rev. B4, 3174 (1971); K. G. Wilson, Rev. Mod. Phys. 47, 1773 (1975); N. Andrei, K. Furuya, and J. H. Lowenstein, Rev. Mod. Phys. 55, 331 (1983).

[11] P. Coleman, Phys. Rev. B28, 5255 (1983); A. J. Millis and P. A. Lee, Phys. Rev. B35, 3394 (1987); N. Read, D. M. Newns, and S. Doniach Phys. Rev. B30, 3841 (1984).

[12] M. A. Continentino and A. P. Malozemoff, Phys. Rev. B33 , 3591 (1986); M.A. Continentino and A.P.Malozemoff, Phys. Rev. B34, 471 (1986).

[13] A. J. Bray and M. A. Moore, J. Phys. C 18, L927 (1985); D. S. Fisher, Phys. Rev. Lett. 56, 416 (1986).

[14] M. A. Continentino, G. M. Japiassu, and A. Troper, Phys. Rev. B 39, 9734 (1989).

[15] M. A. Continentino, J. de Physique I, 693 (1991).

[16] M. A. Continentino, Phys. Rev. B47 , 11587 (1993); M. A. Continentino, G. M. Japiassu and A. Troper, J. Appl. Phys. 73, 6631 (1993)

[17] J. A. Hertz, Phys. Rev. B 14, 1165 (1976).

[18] A. P. Young, J. Phys. C:Solid State Phys. 8, L309 (1975).

[19] M. A. Continentino, Europhysics Letters 9 (1), 77 (1989); M. A. Continentino, Phys. Rev. B42 , 2576 (1990). M. A. Continentino, Phys. Rev. B43 , 6292 (1991); Phys. Rev. B44 , 5397 (1991); M. A. Continentino, Phys. Rev. B45 , 11312 (1992); M. A. Continentino, Physics Reports, 239, 179 (1994); M. A. Continentino and Mauricio D. Coutinho-Filho, Solid State Communications 90 , 619 (1994).

[20] M. P. A. Fisher, P. B. Weichman, G. Grinstein, and D. S. Fisher, Phys. Rev. B40, 546 (1989). 
[21] A. J. Millis, Phys. Rev. B48, 7183 (1993).

[22] A. Aharony, in Critical Phenomena, ed. by Hahne, F.J.W., Springer Verlag, Berlin, 210 (1983).

[23] M. E. Fisher, in Critical Phenomena, ed. by Hahne, F.J.W., Springer Verlag, Berlin, 1 (1983).

[24] M. A. Continentino, Quantum Scaling in Many Body Systems, World Scientific, Singapore, (2001).

[25] M. A. Continentino, Phys. Rev. B55, 5589 (1997).

[26] M. A. Continentino, cond-mat/0408217.

[27] J. G. Sereni, J. Phys. Soc of Japan, 70, 2139 (2001).

[28] J. M. Mignot et al., J. Magn. Magn. Mater. 76 \&77, 97 (1988); A. Lacerda et al., Phys. Rev. B 40, 8759 (1989); J. M. Mignot et al., Phys. Rev. B 40, 10917 (1989); L. Puech et al., J. Low Temp. Phys. 70, 237 (1988). A. Lacerda et al., Phys. Rev. B 40, 11429 (1989); see also references in Ref. [15].

[29] M. A. Continentino, Phys. Rev. B57, 5966 (1998); M. A. Continentino, Physica B 259-261, 172 (1999); M. A. Continentino, Eur. Phys. J. B 13, 31 (2000).

[30] This is different from the theory of Quimiao Si et al., Nature 413, 804 (2001). In our case local criticality is just a regime in the neighborhood of the QCP.

[31] T. Moriya, and T. Takimoto, J. Phys. Soc. Jpn. 64, 960 (1995).

[32] R. Ramazashvili, Phys. Rev. B 60, 7314 (1999); M. A. Continentino, JMMM 272-276 P1, 231 (2004).

[33] A. Schröder et al., Nature, 407, 351 (2000).

[34] K. Kadowaki and S. B. Woods, Solid St. Comm. 58, 507 (1986).

[35] G. R. Stewart, Rev. Mod. Phys. 73, 797 (2001).

[36] H. v. Löhneysen et al., Phys. Rev. Lett. 72, 3262 (1994).

[37] J. Flouquet, Prog. Low Temp. Phys. to be published (2004).
[38] S. Sachdev, Quantum Phase Transitions , Cambridge University Press, UK (1999).

[39] E. Miranda, V. Dobrosavljevic and G. Kotliar, Phys. Rev. Lett. 78, 290 (1997); Chia-Ying Liu, et al., Phys. Rev. B61, 432 (2000); A. H. Castro Neto and B. A. Jones, Phys. Rev. B62, 14975 (2000); M. C. Aronson et al., Phys. Rev. Lett. 75, 725 (1995).

[40] T. G. Rappoport, A. Saguia, B. Boechat, and M. A. Continentino Phys. Rev. B 64, 140402 (2001).

[41] M. A. Continentino and A. S. Ferreira, Physica A339, 461 (2004).

[42] Y. Kitaoka et al., con-mat/0405348.

[43] S. Kawasaki et al., cond-mat/0404376.

[44] A. S. Ferreira, M. A. Continentino and E. C. Marino, Solid State Communications 130, 321 (2004); Physica C 408, 169 (2004) and to be published in Physical Review B.

[45] M. A. Continentino, S. N. de Medeiros, M. T. D. Orlando, M. B. Fontes, E. M. Baggio-Saitovitch, Physical Review B 64, 012404 (2001); J. Larrea et al., submitted to Physical Review Letters.

[46] T. Moriya, Spin Fluctuations in Itinerant Electron Magnetism, Springer-Verlag, Berlin Heidelberg (1985).

[47] E. Bauer, G. Hilscher, H. Michor, Ch. Paul, E. W. Scheidt, A. Gribanov, Yu. Seropegin, H. Noél, M. Sigrist, and P. Rogl, Phys. Rev. Lett. 92, 027003 (2004)

[48] J. Custers, P. Gegenwart, H. Wilhelm, et al., Nature 424, 524 (2003); J. Paglione, M. A. Tanatar, D. G. Hawthorn, E. Boaknin, R. W. Hill, F. Ronning, M. Sutherland, L. Taillefer, C. Petrovic, and P. C. Canfield Phys. Rev. Lett. 91, 246405 (2003) 